Article

\title{
Investigating Potential Chromosomal Rearrangements during Laboratory Culture of Neisseria gonorrhoeae
}

\author{
Russell Spencer-Smith ${ }^{1,2}$, Simon W. Gould ${ }^{1}$, Madhuri Pulijala ${ }^{1}$ and Lori A. S. Snyder ${ }^{1, *}$ (D) \\ 1 School of Life Sciences, Pharmacy, and Chemistry, Kingston University, Penrhyn Road, \\ Kingston upon Thames KT1 2EE, UK; russell.smith@nih.gov (R.S.-S.); S.Gould@kingston.ac.uk (S.W.G.); \\ pretty.madhu@gmail.com (M.P.) \\ 2 National Cancer Institute, National Institutes of Health, 9000 Rockville Pike, Bethesda, MD 20892, USA \\ * Correspondence: L.Snyder@kingston.ac.uk; Tel.: +44-208-417-2003
}

Received: 1 December 2017; Accepted: 19 January 2018; Published: 20 January 2018

\begin{abstract}
Comparisons of genome sequence data between different strains and isolates of Neisseria spp., such as Neisseria gonorrhoeae, reveal that over the evolutionary history of these organisms, large scale chromosomal rearrangements have occurred. Factors within the genomes, such as repetitive sequences and prophage, are believed to have contributed to these observations. However, the timescale in which rearrangements occur is not clear, nor whether it might be expected for them to happen in the laboratory. In this study, $N$. gonorrhoeae was repeatedly passaged in the laboratory and assessed for large scale chromosomal rearrangements. Using gonococcal strain NCCP11945, for which there is a complete genome sequence, cultures were passaged for eight weeks in the laboratory. The resulting genomic DNA was assessed using Pulsed Field Gel Electrophoresis, comparing the results to the predicted results from the genome sequence data. Three cultures generated Pulsed Field Gel Electrophoresis patterns that varied from the genomic data and were further investigated for potential chromosomal rearrangements.
\end{abstract}

Keywords: gonococcus; genome rearrangement; prophage; PFGE; pulsed field gel electrophoresis; genome sequence

\section{Introduction}

Neisseria gonorrhoeae is a strict human pathogen and the causative bacteria of the second most common sexually transmitted infection, gonorrhoea. As of 2008, the complete circularized genome sequence of N. gonorrhoeae strain NCCP11945, from an infected woman in Korea, became the second publically available gonococcal genome [1]. The availability of this second genome sequence led to interesting comparisons between N. gonorrhoeae strains [2-16].

Prior to the genomic era, macrorestriction maps of N. gonorrhoeae strain FA1090 in comparison with the closely related Neisseria meningitidis strain Z2491 showed widespread chromosomal rearrangements between the species [17]. Large scale rearrangements have been shown in silico between genome sequences of N. gonorrhoeae strains FA1090 and NCCP11945 [2]. In comparing these two gonococcal strains, inversions and rearrangements were noted, encompassing much of the genome [2]. Within strain rearrangements have also been noted, including a large inversion of approximately $26 \mathrm{~kb}$ between pilin variants N137 and N138 of N. gonorrhoeae strain MS11, due to recombination between homologous sequences [18].

There are nine identified integrated prophages in N. gonorrhoeae strain FA1090, some of which are complete and active $[19,20]$. There is evidence that ISNgo2 elements within filamentous prophages play a role in large scale genome rearrangements [21]. IS1016 elements also contribute to genome-wide 
rearrangements between N. gonorrhoeae strain FA1090 and N. meningitidis strain Z2491 [22]. Comparison between the genome sequences of N. gonorrhoeae strains FA1090, TCDC-NG08107, and NCCP11945 identified prophages, IS elements, and neisserial Correia Repeat Enclosed Elements (CREE) repeats as mediating the majority of the differences in genomic synteny [10]. Recombination between three lysogenic phages, NGOФ1, NGOФ2, and NGOФ3, resulted in the inversion and rearrangement of sections of the genome with other rearrangements being associated with ISNgo2 elements in filamentous prophages.

Therefore, it appears that regions of homologous DNA, insertion sequences, and integrated prophages have been major driving forces behind chromosomal rearrangements and major contributors to evolution in N. gonorrhoeae. However, this is often based on comparisons between strains and species, investigating the changes in synteny that may have occurred over a relatively long period of evolutionary time. In this study, chromosomal rearrangements were sought after a short period of laboratory culture, in order to determine if the activity of such systems can be seen in currently circulating strains. N. gonorrhoeae strain NCCP11945 was continuously and stressfully cultured over an eight week period. The optimum temperature to cause autolysis in $N$. gonorrhoeae is $40{ }^{\circ} \mathrm{C}$ [23], so cultures were grown at just below this, $39^{\circ} \mathrm{C}$. DNA that is released from lysed gonococcal cells may be taken up and integrated into surviving cells' genomes, contributing to potential rearrangements [24]. The accepted time between passages for $N$. gonorrhoeae is $48 \mathrm{~h}[23,25]$, and therefore, cultures with an increased passage time, $72 \mathrm{~h}$, may result in genomic arrangements. Growth of bacteria in the presence of nalidixic acid has been shown to induce prophages [26,27], so cultures of N. gonorrhoeae with sub-lethal concentrations of nalidixic acid were explored for changes in the genome. Transduction of genetic material by bacteriophages has been demonstrated in N. gonorrhoeae and may have a role in chromosomal changes [24]. Resulting start and endpoint cultures were analyzed by restriction digestion and pulsed field gel electrophoresis (PFGE). These results were compared against next-generation genome sequencing data to investigate the potential presence of large genomic rearrangements between cultures.

\section{Materials and Methods}

\subsection{Growth Conditions}

N. gonorrhoeae strain NCCP11945 [1] was cultured on GC agar plates (Oxoid) with $20 \mu \mathrm{M}$ iron III nitrate and a glucose-based nutritional supplement [25] in a culture jar with $\mathrm{CO}_{2}$ Gen sachets (Oxoid). GC broth was formulated as previously [28]. Preliminary tests of growth conditions determined that N. gonorrhoeae strain NCCP11945 was able to grow at $41^{\circ} \mathrm{C}$, but was unsustainable through repeated passage, and therefore $39^{\circ} \mathrm{C}$ was selected as the temperature stress for eight weeks' passage. Likewise, repeated passage at four days was not sustainable, and therefore three-day passage was used for nutrient stress. Sub-lethal nalidixic acid concentrations were established by growing N. gonorrhoeae strain NCCP11945 on GC agar containing 2, 4, 8, 16, 32, 64, 128, and $256 \mu \mathrm{g} / \mathrm{mL}$ nalidixic acid. Although $256 \mu \mathrm{g} / \mathrm{mL}$ nalidixic acid permitted a low level of growth, it was again not sustainable through repeated passages and $128 \mu \mathrm{g} / \mathrm{mL}$ nalidixic acid was used as the prophage inducing stress. Viable cultures under three conditions $\left(39^{\circ} \mathrm{C}\right.$, passage each three days, and with $128 \mu \mathrm{g} / \mathrm{mL}$ nalidixic acid) and a control ( $37^{\circ} \mathrm{C}$ and passage each two days) were maintained in duplicate for a period of eight weeks. At each passage, a sweep of growth on the plates was resuspended in GC broth and used to inoculate the next plate, thereby avoiding potential bias via the selection of individual colonies. Growth was determined visually based on colony size and number observed.

\subsection{Pulsed Field Gel Electrophoresis}

Start-point and end-point samples from the cultures were grown overnight in triplicate and the growth was resuspended in GC broth, embedded in agarose, and DNA extracted, as per the Bio-Rad CHEFII Plug Kit protocol. The agarose plugs were restriction digested with 20 U SpeI (Fermentas, 
Loughborough, UK) for $16 \mathrm{~h}$ at $37^{\circ} \mathrm{C}$. SpeI digested plugs were loaded onto a $1 \%$ agarose gel with flanking PFG lambda size markers (New England Biolabs, Hithchin, UK). The SpeI PFGE was run on a Bio-Rad CHEFII pulsed field system at $150 \mathrm{~V}$ in $0.5 \times \mathrm{TBE}$ buffer at $15^{\circ} \mathrm{C}$ for $48 \mathrm{~h}$ with pulse times ramped from $5 \mathrm{~s}$ initially to $120 \mathrm{~s}$ final time. BglII digested plugs were loaded onto a $1 \%$ agarose gel with flanking PFG low-range size markers (New England Biolabs, Hitchin, UK) and run on a Bio-Rad (Watford, UK) CHEFII pulsed field rig at $200 \mathrm{~V}$ in $0.5 \times$ TBE buffer at $15^{\circ} \mathrm{C}$ for $24 \mathrm{~h}$ with pulse times ramped from $1 \mathrm{~s}$ initially to $25 \mathrm{~s}$ final time. All gels were stained in a $1 \mu \mathrm{M}$ solution of ethidium bromide for $45 \mathrm{~min}$, washed in $\mathrm{dH}_{2} \mathrm{O}$ for $45 \mathrm{~min}$, and visualized under UV light.

\subsection{Predicted PFGE Band Patterns}

The complete genome sequence data from N. gonorrhoeae strain NCCP11945 [1] was analysed for the presence of SpeI restriction enzyme recognition sites, $5^{\prime}$-ACTAGT-3' (Table S1), and BglII restriction enzyme recognition sites, $5^{\prime}$-AGATCT-3' (Table S2), using the Sequence Manipulation Suite: DNA Pattern Find [29]. These enzymes were chosen based on previous PFGE and mapping experiments in other $N$. gonorrhoeae strains $[30,31]$. The predicted fragment sizes and their locations in the genome sequence [1] were noted and mapped to the chromosome using DNAPlotter [32] (Figure 1).

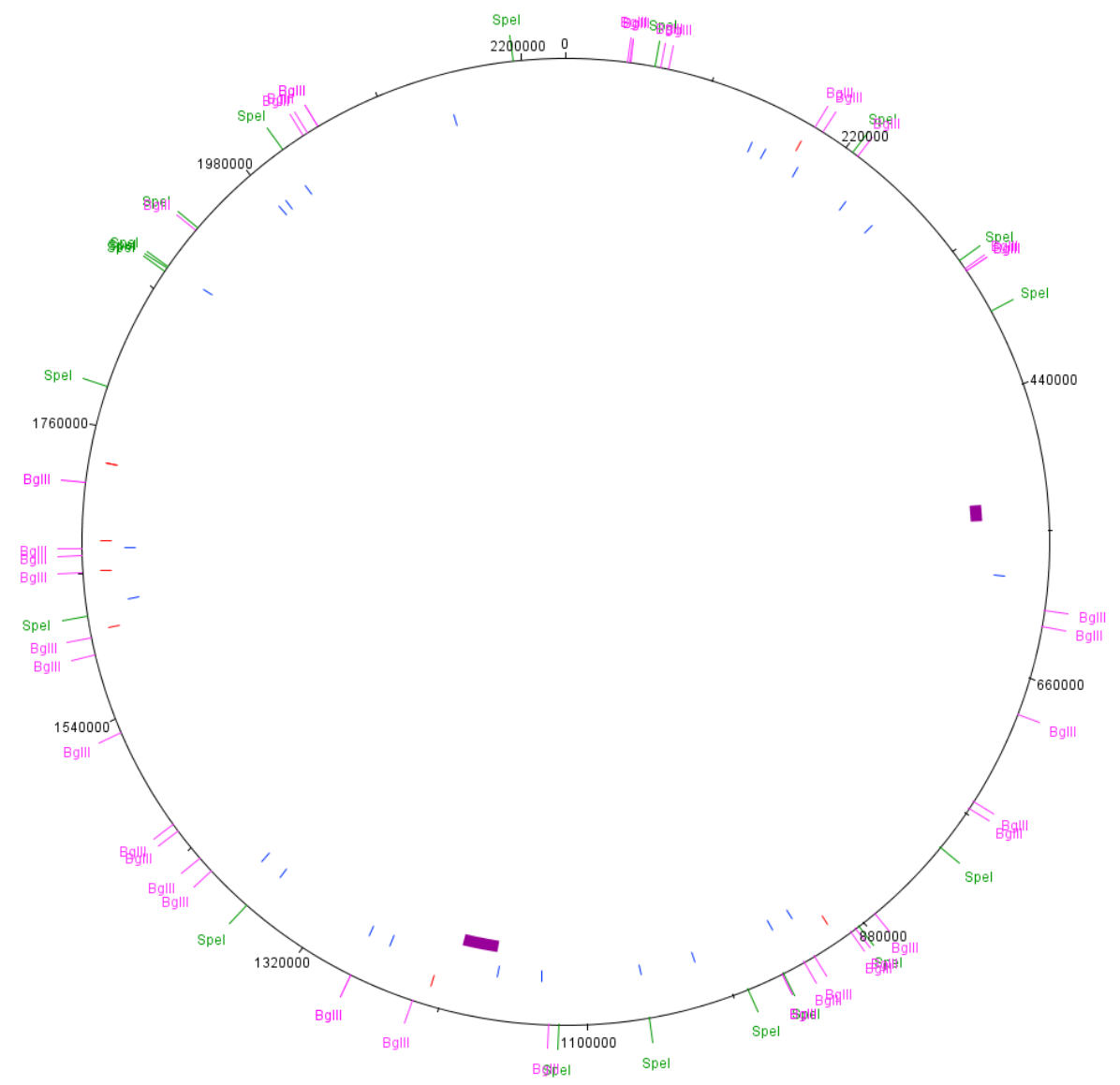

Figure 1. Chromosomal map of N. gonorrhoeae strain NCCP11945. Each of the identified restriction digest sites was mapped to the chromosome of N. gonorrhoeae strain NCCP11945: SpeI shown in green and $B g I I I$ in pink. In red are locations of ISNgo2 elements, in blue are IS1016, and purple blocks represent the locations of the NGOФ1.

\subsection{Next Generation Genome Sequencing}

Cultures were grown for eight weeks under standard conditions and for eight weeks with sub-lethal $128 \mu \mathrm{g} / \mathrm{mL}$ nalidixic acid. DNA extraction used the Gentra Puregene Yeast/Bact. Kit (Qiagen, Manchester, UK); $1 \mu \mathrm{g}$ of the extracted DNA was genome sequenced on the Life Technologies 
Ion Personal Genome Machine using the Ion Express Fragment Library kit, Ion Express Template kit, and Ion Sequencing kit (Life Technologies, Loughborough, UK). Generated sequence data was mapped to the reference sequence data (CP001050; [1]) using Galaxy. The Ion Torrent bam format files were converted to fastq format using BAMTools Convert [33]. FASTQ Groomer was used on all NGS data [34]. Bowtie2 was used to map the reads against the reference $[35,36]$ before visualization using the Integrated Genomics Viewer (IGV) [37,38]. Each restriction enzyme digest site was manually analysed for SNPs or other changes to the recognition sequence. Mapped reads were assessed manually in IGV for split reads at suspected chromosomal breakpoints. De novo assembly of unmapped reads used Unicycler [39]. Data is available from NCBI SRA SRR3547950 (standard) and SRR4431963 (nalidixic acid).

\section{Results}

\subsection{Growth of Bacteria in the Laboratory under Different Conditions}

Differences in the growth of the N. gonorrhoeae strain NCCP11945 in the four culture conditions $\left(37^{\circ} \mathrm{C}, 39^{\circ} \mathrm{C}\right.$, three-day passage, $128 \mu \mathrm{g} / \mathrm{mL}$ nalidixic acid) initially resulted in observable differences between the cultures. $N$. gonorrhoeae could not be successfully repeatedly passaged at $41{ }^{\circ} \mathrm{C}$, but was successful at $39^{\circ} \mathrm{C}$. N. gonorrhoeae strain NCCP11945 grew at 2, 4, 8, 16, 32, 64, 128, and $256 \mu \mathrm{g} / \mathrm{mL}$ nalidixic acid, but the growth on $256 \mu \mathrm{g} / \mathrm{mL}$ nalidixic acid was not sustainable. Passage each four days likewise resulted in a failure of the culture to grow, whilst passage each three days remained viable. Thus, cultures were propagated for eight weeks under control conditions, $39^{\circ} \mathrm{C}$, three-day passage, and with $128 \mu \mathrm{g} / \mathrm{mL}$ nalidixic acid.

Control cultures grown at $37^{\circ} \mathrm{C}$ and passaged to fresh media every two days showed consistent growth throughout the eight-week period. Cultures passaged every three days had approximately $20 \%$ more colonies on the third day compared to day two. Although there was more growth on day three than day two, the rate of growth on the plate appeared lower than seen for the previous two days. Initial growth for the $39{ }^{\circ} \mathrm{C}$ cultures was approximately $50 \%$ fewer colonies than observed in the control cultures; however, the size of individual colonies was larger. Successive passages at $39^{\circ} \mathrm{C}$ increased the colony number to a level comparable to or greater than the control. Nalidixic acid containing cultures initially showed approximately $40 \%$ fewer colonies, which were approximately $20 \%$ smaller than those of the control culture. By week two, the colony size and number was equivalent to that of control cultures.

\subsection{Pulsed Field Gel Electrophoresis}

Both SpeI digestion and BglII digestion produced a majority of reproducible bands across all experiments (Figures 2 and 3). These results correlated well with the expected band sizes predicted from the reference genome sequence [1].

\subsubsection{SpeI Digestion and PFGE}

PFGE of SpeI digested of agarose plugs containing N. gonorrhoeae strain NCCP11945 DNA, produced bands that are present in all of the culture conditions and the starting culture (Figure 2). There is an additional band of approximately $500 \mathrm{~kb}$ in the lanes for the control culture and the culture passaged every three days (nutrient stress) that is not evident in the other lanes. The lane for the culture grown on $128 \mu \mathrm{g} / \mathrm{mL}$ nalidixic acid shows an additional band around $210 \mathrm{~kb}$.

\subsubsection{BglII Digestion and PFGE}

The PFGE of the BglII digestions showed bands that are clearly present in all of the lanes, representing the different cultures and their starting inoculum (Figure 3). The culture that was passaged every three days has an additional band of around $168 \mathrm{~kb}$ and the almost complete absence of the band of approximately $130 \mathrm{~kb}$ (Figure 3). 


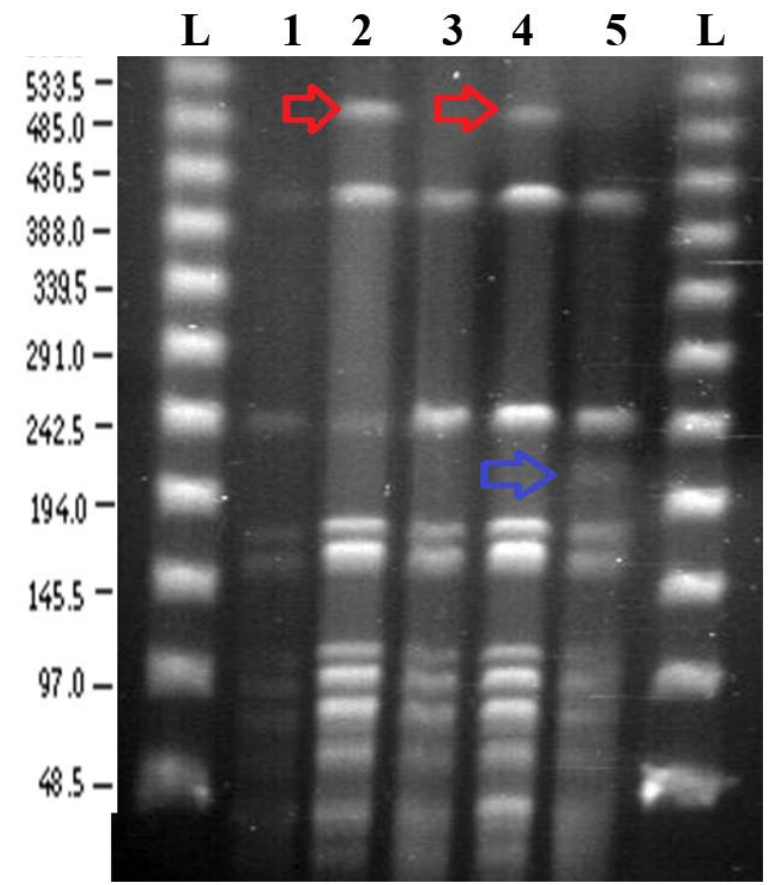

Figure 2. PFGE gel image of SpeI digested N. gonorrhoeae strain NCCP11945 cultures. L: NEB lambda ladders; 1 : temperature stress culture grown at $39{ }^{\circ} \mathrm{C}$ for eight weeks; 2 : control culture; 3: starting inoculum; 4: nutrient stress culture passaged every three days; 5 : prophage stress culture grown with $125 \mu \mathrm{g} / \mathrm{mL}$ nalidixic acid. Coloured arrows represent bands that are present only in those lanes: red arrows, about $500 \mathrm{~kb}$; blue arrow, about $210 \mathrm{~kb}$.

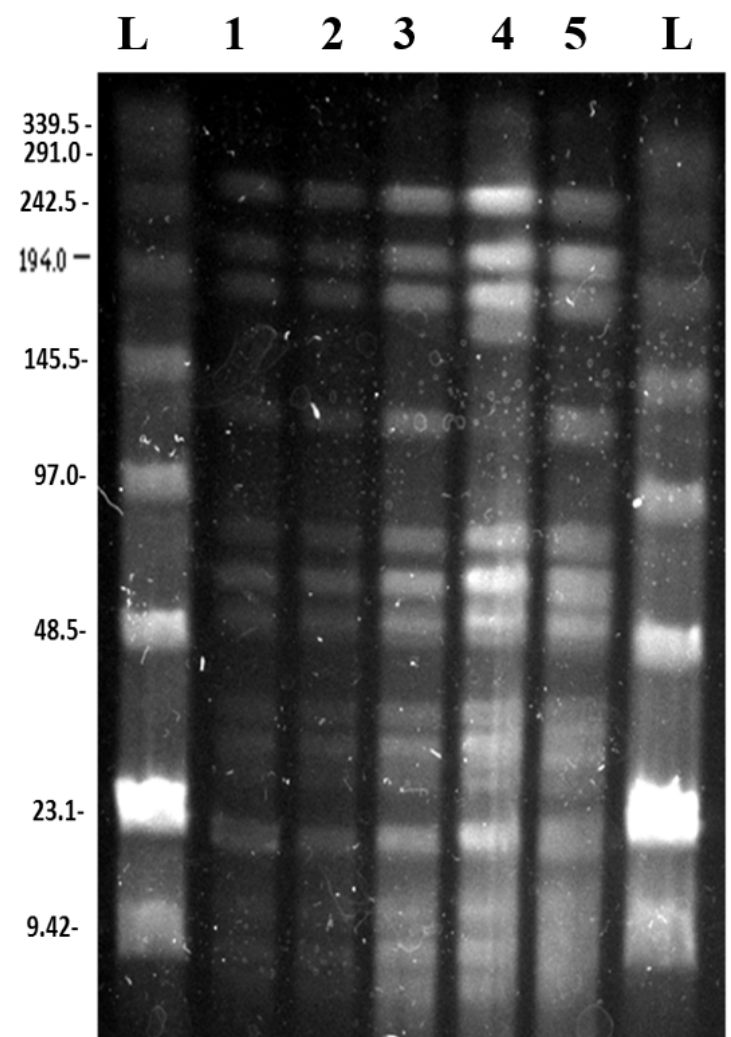

Figure 3. PFGE gel image of BglII digested N. gonorrhoeae strain NCCP11945 cultures. L: NEB lambda ladders; 1 : temperature stress culture grown at $39{ }^{\circ} \mathrm{C}$ for eight weeks; 2 : control culture; 3 : starting inoculum; 4: nutrient stress culture passaged every three days; 5 : prophage stress culture grown with $125 \mu \mathrm{g} / \mathrm{mL}$ nalidixic acid. 


\subsection{Predicted PFGE Band Patterns Based on Genome Sequence Data}

Based on the whole genome sequence of N. gonorrhoeae strain NCCP11945 [1], the restriction digest sites were identified and fragment sizes predicted for SpeI (Table S1) and BglII (Table S2). Comparison between this in silico data and the experimental data (Figures 2 and 3) demonstrates that the assembly of the N. gonorrhoeae strain NCCP11945 genome sequence is robust. All of the genome sequence SpeI predicted fragment sizes that could be resolved on the PFGE are present and the majority of the $B g l$ II bands are present aside from a region of the gel around $110,000 \mathrm{bp}$, where no bands are readily visible and BglII fragments are too small to be resolved. From the BglII PFGE, it was observed that there was almost a complete absence of the band at approximately 130,000 bp in the nutrient stress culture (Figure 3). This corresponds to the 131,206 bp band predicted from the genome sequence data (Table S2).

\subsection{Comparison of PFGE Results with Genome Sequence Data from the Cultures}

Ion Torrent next generation sequencing of the control and prophage stress cultures was undertaken to determine the cause(s) of the additional bands observed on the SpeI PFGE. This data, mapped to the N. gonorrhoeae strain NCCP11945 genome sequence (CP001051.1), showed that the SpeI and BglII restriction endonuclease recognition sequences (Tables S1 and S2) have not changed in the cultures. Therefore, differences in the PFGE bands from those expected are not due to mutation of the digest sites.

Focusing on the sequence data from the prophage stress culture, where an additional band was observed in the SpeI PFGE, the mapping of the sequence reads to the reference genome did not reveal any large regions of deletion. De novo assembly of the unaligned reads revealed that all unaligned reads were part of the pNGK plasmid present within N. gonorrhoeae strain NCCP11945.

\section{Discussion}

Continuous passage of $N$. gonorrhoeae in the laboratory produced some changes in the growth phenotype. Culturing of N. gonorrhoeae strain NCCP11945 at $41{ }^{\circ} \mathrm{C}$ could not be sustained (data not shown); however, the bacteria were able to adapt to and thrive at $39{ }^{\circ} \mathrm{C}$. This suggests that $N$. gonorrhoeae is able to survive fevers, such as those associated with gonococcal arthritis; however, a lack of prolonged growth at $41^{\circ} \mathrm{C}$ is likely the origin of reported success with artificial fever therapy treatments in the past [40].

PFGE from the cultures showed a majority of reproducible bands that correlated to the predicted band sizes from genomic data. An additional $500 \mathrm{~kb}$ fragment was seen in the SpeI digest of the control and nutrient stress cultures (Figure 2, lanes 2 and 4). A mutation in the restriction site at genomic position 1,371,473 would join the 250,422 bp and 247,231 bp (Table S1) fragments, making a fragment of 497,653 bp. However, genome sequence data from this sample did not indicate a mutation in this restriction site. A large PFGE band of about $500 \mathrm{~kb}$ in SpeI digests of N. gonorrhoeae strain MS11-N198 has been previously reported [30]. It was determined that this SpeI digest site becomes resistant to digestion in 10-90\% of the gonococcal population [30]. This is consistent with our results for N. gonorrhoeae strain NCCP11945 (Figure 2), where the equivalent SpeI digest site to the resistant site in strain MS11-N198 is that at position 1,371,473 in strain NCCP11945 (Figure 1). This also explains why the $\sim 500 \mathrm{~kb}$ band is not visible in the other PFGE lanes.

A SpeI PFGE fragment of around $210 \mathrm{~kb}$ is grown on a sub-lethal concentration of nalidixic acid in the culture (Figure 2, lane 5). Unlike the $500 \mathrm{~kb} \mathrm{SpeI} \mathrm{fragment,} \mathrm{there} \mathrm{are} \mathrm{no} \mathrm{two} \mathrm{adjoining}$ SpeI fragments which equal around $210 \mathrm{~kb}$. The additional band may therefore be the result of rearrangement. The chromosomes of N. gonorrhoeae strain NCCP11945 and strain FA1090 differ in the presence of the Gonococcal Genetic Island [41,42] and in inversions mediated by CREE [2] and SSREE sequences [10]. Inversions or excisions of these elements cannot account for the generation of the $210 \mathrm{~kb}$ PFGE band. 
SpeI fragment 250,422 bp contains NGOФ1, NGOФ2, NGOФ3, and NGOФ7 and fragment 247,231 bp contains NGOФ2, NGOФ3, and NGOФ8. Both include ISNgo2 elements, which have been shown to be the driving factor in several large scale rearrangements in N. gonorrhoeae [21]. However, inversions or excisions between these or other ISNgo2 elements in the chromosome $[10,19]$ would also not generate the observed $210 \mathrm{~kb}$ fragment. The mapped sequence data has lower coverage in the regions containing these prophages, with some regions of up to 200 bases having no coverage. De novo assembly of the genomic data and alignment with the reference genome suggests that there are deletions and rearrangements in these regions, although the presence of multiple copies of the prophage sequences may have generated misassemblies. NGOФ2 has been shown to be functional [20], and therefore it is possible that a prophage mediated process has caused changes in the $N$. gonorrhoeae genome in the presence of nalidixic acid, known to activate prophage gene expression. The NGOФ1 within the 250,422 SpeI fragment is 30,030 bp, and therefore, the loss of this prophage would result in a band of 220,392 bp (Figure 1). The precise identity of the additional band on the PFGE cannot be determined given the data; however, it is likely to be due to NGO $\Phi 1$ or NGOФ2. There is a high degree of sequence similarity between the NGOФ1, NGOФ2, and NGOФ3 prophages [20], complicating the mapping of sequence reads and the de novo assembly and alignment of sequences against the reference genome. Therefore, there is no solid sequencing data supporting the mobilisation of prophages. The additional $210 \mathrm{~kb}$ band in the nalidixic acid culture is either an experimental artefact occurring in just one lane or the result of changes to the genome via the activity of the neisserial prophage. The latter would be expected under these culture conditions; however, any resulting chromosomal rearrangements would need to be determined using an extra-long sequencing read technology, such as the Oxford Nanopore or PacBio systems.

The BglII PFGE of the nutrient stress culture has an additional band of approximately 168,000 bp and the corresponding almost complete absence of the 131,206 bp band (Figure 3, lane 4). These bands cannot be accounted for through the loss of a BglII site. Given the sequence results from the control and nalidixic acid-containing culture, sequencing did not clearly elucidate the cause of the additional PFGE bands and the absence of a range of bands on the BglII PFGE, and the nutrient stress culture was not sequenced.

It is evident in the sequencing data that small changes associated with phase variable changes in repeat tracts and inversion do occur, as have been observed and reported previously [16,43]. The growth culture phenotypes observed, where the stress cultures grow less well than the control initially and then adapt (Tables S1 and S2), are likely to be due to phase variable switching of gene expression. Phase variation is known to be involved in stochastic switching of gene expression that confers an adaptive advantage over the other cells in the culture [16,44-47]. Therefore, the observed changes in the phenotype and small changes in the genome sequence data concur with previous studies. De novo assembly of the sequence data and alignment with the reference genome suggests that there may be larger scale rearrangements; however, these could be misassemblies and did not correspond to data from mapped reads. Contigs can be generated that suggest that there are large rearrangements, but without long sequence read data such changes cannot be confirmed.

\section{Conclusions}

Pulsed field gel electrophoresis can confirm the order of assembly of next generation genome sequence data. Using the final circularized assembly of a bacterial genome sequence, the PFGE digest pattern can be predicted and compared to the in silico data. Over time, changes in the genome can occur in the laboratory. Here, we show that N. gonorrhoeae strain NCCP11945 was able to grow at $41{ }^{\circ} \mathrm{C}$, to grow after four days of growth, and to grow on media with $256 \mu \mathrm{g} / \mathrm{mL}$ nalidixic acid; however, growth in these conditions was not sustainable. Observable differences in growth resolved by three weeks in conditions of $39^{\circ} \mathrm{C}$, after passage each three days, and in media with $128 \mu \mathrm{g} / \mathrm{mL}$ nalidixic acid. These adaptations are likely to be due to small genetic changes, such as phase variable gene expression [16,43], which are evident in corresponding sequence data. 
Potential large scale chromosomal rearrangements suggested by additional bands on PFGE are due to a previously reported resistant restriction enzyme digest site [30] and potential filamentous prophage induction and transposition. It is clear that investigations into the genotypic origins of phenotypic changes in N. gonorrhoeae are complex, involving phase variable changes, changes in restriction site sensitivity, and rearrangements mediated by prophage and prophage associated elements, all of which may dynamically change in culture, contributing to population diversity.

Supplementary Materials: The following are available online at http://www.mdpi.com/2076-2607/6/1/10/s1. Acknowledgments: This work was funded by a Kingston University studentship to R.S.-S.

Author Contributions: R.S.-S. and L.A.S.S. conceived and designed the experiments; R.S.-S., S.W.G., and M.P. performed the experiments; R.S.-S., M.P., and L.A.S.S. analyzed the data; S.W.G. contributed reagents and equipment; L.A.S.S. and R.S.-S. wrote the paper.

Conflicts of Interest: The authors declare no conflict of interest.

\section{References}

1. Chung, G.T.; Yoo, J.S.; Oh, H.B.; Lee, Y.S.; Cha, S.H.; Kim, S.J.; Yoo, C.K. Complete genome sequence of Neisseria gonorrhoeae NCCP11945. J. Bacteriol. 2008, 190, 6035-6036. [CrossRef] [PubMed]

2. Snyder, L.A.; Cole, J.A.; Pallen, M.J. Comparative analysis of two Neisseria gonorrhoeae genome sequences reveals evidence of mobilization of Correia Repeat Enclosed Elements and their role in regulation. BMC Genomics 2009, 10, 70. [CrossRef] [PubMed]

3. Bennett, J.S.; Bentley, S.D.; Vernikos, G.S.; Quail, M.A.; Cherevach, I.; White, B.; Parkhill, J.; Maiden, M.C. Independent evolution of the core and accessory gene sets in the genus Neisseria: Insights gained from the genome of Neisseria lactamica isolate 020-06. BMC Genomics 2010, 11, 652. [CrossRef] [PubMed]

4. Ohnishi, M.; Watanabe, Y.; Ono, E.; Takahashi, C.; Oya, H.; Kuroki, T.; Shimuta, K.; Okazaki, N.; Nakayama, S.; Watanabe, H. Spread of a chromosomal cefixime-resistant penA gene among different Neisseria gonorrhoeae lineages. Antimicrob. Agents Chemother. 2010, 54, 1060-1067. [CrossRef] [PubMed]

5. Anderson, M.T.; Seifert, H.S. Opportunity and means: Horizontal gene transfer from the human host to a bacterial pathogen. MBio 2011, 2, e00005-11. [CrossRef] [PubMed]

6. Chen, C.C.; Hsia, K.C.; Huang, C.T.; Wong, W.W.; Yen, M.Y.; Li, L.H.; Lin, K.Y.; Chen, K.W.; Li, S.Y. Draft genome sequence of a dominant, multidrug-resistant Neisseria gonorrhoeae strain, TCDC-NG08107, from a sexual group at high risk of acquiring human immunodeficiency virus infection and syphilis. J. Bacteriol. 2011, 193, 1788-1789. [CrossRef] [PubMed]

7. De Curraize, C.; Kumanski, S.; Micaëlo, M.; Fournet, N.; La Ruche, G.; Meunier, F.; Amarsy, R.; Jacquier, H.; Cambau, E.; Goubard, A.; et al. Ceftriaxone-resistant Neisseria gonorrhoeae isolates (2010 to 2014) in France characterized by using whole-genome sequencing. Antimicrob. Agents Chemother. 2016, 60, 6962-6964. [CrossRef] [PubMed]

8. Lin, Y.H.; Ryan, C.S.; Davies, J.K. Neisserial Correia repeat-enclosed elements do not influence the transcription of pil genes in Neisseria gonorrhoeae and Neisseria meningitidis. J. Bacteriol. 2011, 193, 5728-5736. [CrossRef] [PubMed]

9. Bennett, J.S.; Jolley, K.A.; Earle, S.G.; Corton, C.; Bentley, S.D.; Parkhill, J.; Maiden, M.C. A genomic approach to bacterial taxonomy: An examination and proposed reclassification of species within the genus Neisseria. Microbiology 2012, 158, 1570-1580. [CrossRef] [PubMed]

10. Spencer-Smith, R.; Varkey, E.M.; Fielder, M.D.; Snyder, L.A. Sequence features contributing to chromosomal rearrangements in Neisseria gonorrhoeae. PLoS ONE 2012, 7, e46023. [CrossRef] [PubMed]

11. Guo, F.B.; Xiong, L.; Teng, J.L.; Yuen, K.Y.; Lau, S.K.; Woo, P.C. Re-annotation of protein-coding genes in 10 complete genomes of Neisseriaceae family by combining similarity-based and composition-based methods. DNA Res. 2013, 20, 273-286. [CrossRef] [PubMed]

12. Putonti, C.; Nowicki, B.; Shaffer, M.; Fofanov, Y.; Nowicki, S. Where does Neisseria acquire foreign DNA from: An examination of the source of genomic and pathogenic islands and the evolution of the Neisseria genus. BMC Evol. Biol. 2013, 13, 184. [CrossRef] [PubMed] 
13. Jamet, A.; Jousset, A.B.; Euphrasie, D.; Mukorako, P.; Boucharlat, A.; Ducousso, A.; Charbit, A.; Nassif, X. A new family of secreted toxins in pathogenic Neisseria species. PLoS Pathog. 2015, 11, e1004592. [CrossRef] [PubMed]

14. McClure, R.; Nudel, K.; Massari, P.; Tjaden, B.; Su, X.; Rice, P.A.; Genco, C.A. The Gonococcal Transcriptome during Infection of the Lower Genital Tract in Women. PLoS ONE 2015, 10, e0133982. [CrossRef] [PubMed]

15. Spencer-Smith, R.; Roberts, S.; Gurung, N.; Snyder, L.A.S. DNA uptake sequences in Neisseria gonorrhoeae as intrinsic transcriptional terminators and markers of horizontal gene transfer. Microb. Genomics 2016, 2, e000069. [CrossRef] [PubMed]

16. Zelewska, M.A.; Pulijala, M.; Spencer-Smith, R.; Mahmood, H.A.; Norman, B.; Churchward, C.P.; Calder, A.; Snyder, L.A. Phase variable DNA repeats in Neisseria gonorrhoeae influence transcription, translation, and protein sequence variation. Microb. Genomics 2016, 2, e000078. [CrossRef] [PubMed]

17. Dempsey, J.A.; Wallace, A.B.; Cannon, J.G. The physical map of the chromosome of a serogroup A strain of Neisseria meningitidis shows complex rearrangements relative to the chromosomes of the two mapped strains of the closely related species N. gonorrhoeae. J. Bacteriol. 1995, 177, 6390-6400. [CrossRef] [PubMed]

18. Gibbs, C.P.; Meyer, T.F. Genome plasticity in Neisseria gonorrhoeae. FEMS Microbiol. Lett. 1996, 145, $173-179$. [CrossRef] [PubMed]

19. Kawai, M.; Uchiyama, I.; Kobayashi, I. Genome comparison in silico in Neisseria suggests integration of filamentous bacteriophages by their own transposase. DNA Res. 2005, 12, 389-401. [CrossRef] [PubMed]

20. Piekarowicz, A.; Klyz, A.; Majchrzak, M.; Adamczyk-Poplawska, M.; Maugel, T.K.; Stein, D.C. Characterization of the dsDNA prophage sequences in the genome of Neisseria gonorrhoeae and visualization of productive bacteriophage. BMC Microbiol. 2007, 7, 66. [CrossRef] [PubMed]

21. Skaar, E.P.; LeCuyer, B.; Lenich, A.G.; Lazio, M.P.; Perkins-Balding, D.; Seifert, H.S.; Karls, A.C. Analysis of the Piv recombinase-related gene family of Neisseria gonorrhoeae. J. Bacteriol. 2005, 187, 1276-1286. [CrossRef] [PubMed]

22. Kawai, M.; Nakao, K.; Uchiyama, I.; Kobayashi, I. How genomes rearrange: Genome comparison within bacteria Neisseria suggests roles for mobile elements in formation of complex genome polymorphisms. Gene 2006, 383, 52-63. [CrossRef] [PubMed]

23. Hebeler, B.H.; Young, F.E. Autolysis of Neisseria gonorrhoeae. J. Bacteriol. 1976, 122, 385-392.

24. Cehovin, A.; Lewis, S.B. Mobile genetic elements in Neisseria gonorrhoeae: Movement for change. Pathog. Dis. 2017, 75, ftx071. [CrossRef] [PubMed]

25. Kellogg, D.S.; Peacock, W.L.; Deacon, W.E.; Brown, L.; Pirkle, C.I. Neisseria gonorrhoeae I: Virulence genetically linked to clonal variation. J. Bacteriol. 1963, 85, 1274-1279. [PubMed]

26. Cowlishaw, J.; Ginoza, W. Induction of lambda prophage by nalidixic acid. Virology 1970, 41, $244-255$. [CrossRef]

27. Vihanová, D.; Vondrejs, V. Induction of bacteriophage lambda with oxolinic and nalidixic acid. Folia Microbiol. (Praha) 1974, 19, 390-393. [CrossRef] [PubMed]

28. Qu, X.-D.; Harwig, S.S.L.; Oren, A.; Shafer, W.M.; Lehrer, R.I. Susceptibility of Neisseria gonorrhoeae to protegrins. Infect. Immun. 1996, 64, 1240-1245. [PubMed]

29. Stothard, P. The Sequence Manipulation Suite: JavaScript programs for analyzing and formatting protein and DNA sequences. Biotechniques 2000, 28, 1102-1104. [PubMed]

30. Bihlmaier, A.; Römlin, U.; Meyer, T.F.; Tümmler, B.; Gibbs, C.P. Physical and genetic map of the Neisseria gonorrhoeae strain MS11-N198 chromosome. Mol. Microbiol. 1991, 5, 2529-2539. [CrossRef]

31. Dempsey, J.A.; Litaker, W.; Madhure, A.; Snodgrass, T.L.; Cannon, J.G. Physical map of the chromosome of Neisseria gonorrhoeae FA1090 with locations of genetic markers, including opa and pil genes. J. Bacteriol. 1991, 173, 5476-5486. [CrossRef] [PubMed]

32. Carver, T.; Thomson, N.; Bleasby, A.; Berriman, M.; Parkhill, J. DNAPlotter: Circular and linear interactive genome visualization. Bioinformatics 2009, 25, 119-120. [CrossRef] [PubMed]

33. Barnett, D.W.; Garrison, E.K.; Quinlan, A.R.; Strömberg, M.P.; Marth, G.T. BamTools: A C++ API and toolkit for analyzing and managing BAM files. Bioinformatics 2011, 27, 1691-1692. [CrossRef] [PubMed]

34. Blankenberg, D.; Gordon, A.; Von Kuster, G.; Coraor, N.; Taylor, J.; Nekrutenko, A.; Galaxy Team. Manipulation of FASTQ data with Galaxy. Bioinformatics 2010, 26, 1783-1785. [CrossRef] [PubMed]

35. Langmead, B.; Trapnell, C.; Pop, M.; Salzberg, S.L. Ultrafast and memory-efficient alignment of short DNA sequences to the human genome. Genome Biol. 2009, 10, R25. [CrossRef] [PubMed] 
36. Langmead, B.; Salzberg, S.L. Fast gapped-read alignment with Bowtie 2. Nat. Methods 2012, 9, $357-359$. [CrossRef] [PubMed]

37. Robinson, J.T.; Thorvaldsdóttir, H.; Winckler, W.; Guttman, M.; Lander, E.S.; Getz, G.; Mesirov, J.P. Integrative genomics viewer. Nat. Biotechnol. 2011, 29, 24-26. [CrossRef] [PubMed]

38. Thorvaldsdóttir, H.; Robinson, J.T.; Mesirov, J.P. Integrative Genomics Viewer (IGV): High-performance genomics data visualization and exploration. Brief. Bioinform. 2013, 14, 178-192. [CrossRef] [PubMed]

39. Wick, R.R.; Judd, L.M.; Gorrie, C.L.; Holt, K.E. Unicycler: Resolving bacterial genome assemblies from short and long sequencing reads. PLoS Comput. Biol. 2017, 13, e1005595. [CrossRef] [PubMed]

40. Robinson, J.A.; Hirsh, H.L.; Zeller, W.W.; Dowling, H.F. Gonococcal arthritis; a study of 202 patients treated with penicillin, sulfonamides or fever therapy. Ann. Intern. Med. 1949, 30, 1212-1223. [PubMed]

41. Dillard, J.P.; Seifert, H.S. A variable genetic island specific for Neisseria gonorrhoeae is involved in providing DNA for natural transformation and is found more often in disseminated infection isolates. Mol. Microbiol. 2001, 41, 263-277. [CrossRef] [PubMed]

42. Snyder, L.A.; Jarvis, S.A.; Saunders, N.J. Complete and variant forms of the 'gonococcal genetic island' in Neisseria meningitidis. Microbiology 2005, 151, 4005-4013. [CrossRef] [PubMed]

43. Elbeyioglu, F.; Roberts, S.B.; Spencer-Smith, R.; Pulijala, M.; Zelewska, M.A.; Nebel, J.C.; Snyder, L.A. Inversion of Correia Repeat Enclosed Elements in Neisseria gonorrhoeae. Microbiology 2016, 163, 31-36. [CrossRef] [PubMed]

44. Zöllner, R.; Oldewurtel, E.R.; Kouzel, N.; Maier, B. Phase and antigenic variation govern competition dynamics through positioning in bacterial colonies. Sci. Rep. 2017, 7, 12151. [CrossRef] [PubMed]

45. Klughammer, J.; Dittrich, M.; Blom, J.; Mitesser, V.; Vogel, U.; Frosch, M.; Goesmann, A.; Müller, T.; Schoen, C. Comparative genome sequencing reveals within-host genetic changes in Neisseria meningitidis during invasive disease. PLoS ONE 2017, 12, e0169892. [CrossRef] [PubMed]

46. Cole, J.G.; Fulcher, N.B.; Jerse, A.E. Opacity proteins increase Neisseria gonorrhoeae fitness in the female genital tract due to a factor under ovarian control. Infect. Immun. 2010, 78, 1629-1641. [CrossRef] [PubMed]

47. Snyder, L.A.; Butcher, S.A.; Saunders, N.J. Comparative whole-genome analyses reveal over 100 putative phase-variable genes in the pathogenic Neisseria spp. Microbiology 2001, 147, 2321-2332. [CrossRef] [PubMed]

(C) 2018 by the authors. Licensee MDPI, Basel, Switzerland. This article is an open access article distributed under the terms and conditions of the Creative Commons Attribution (CC BY) license (http:/ / creativecommons.org/licenses/by/4.0/). 